\title{
Factors Influencing Maternal Adherence to Infant's Nevirapine Prophylaxis in a Cross-Sectional Study Conducted at Mulago Hospital, Kampala, Uganda
}

\author{
Caroline Kambugu Nabasirye ${ }^{1}$, Ratib Mawa ${ }^{2}$, Elizabeth Ayebare ${ }^{3}$ \\ ${ }^{1}$ Department of Nursing and Midwifery, Faculty of Health Sciences, Lira University, Lira, Uganda \\ ${ }^{2}$ Department of Public Health, Faculty of Health Sciences, Victoria University, Kampala, Uganda \\ ${ }^{3}$ Department of Nursing, School of Health Sciences, Makerere University College of Health Sciences, Kampala, Uganda
}

Email address:

leakycarolyn326@gmail.com (C. K. Nabasirye)

\section{To cite this article:}

Caroline Kambugu Nabasirye, Ratib Mawa, Elizabeth Ayebare. Factors Influencing Maternal Adherence to Infant's Nevirapine Prophylaxis Regimen in Cross-Sectional Study Conducted at MulagoNational Referral Hospital, Kampala, Uganda.International Journal of HIV/AIDS Prevention, Education and Behavioural Science. Vol. 5, No. 1, 2019, pp. 37-46. doi: 10.11648/j.ijhpebs.20190501.15

Received: February 14, 2019; Accepted: April 3, 2019; Published: May 6, 2019

\begin{abstract}
To eliminate mother to child transmission of HIV, existing interventions might need to be scaled-up and rigorously implemented. One of the strategies to reduce vertical transmission of HIVis the 4-6weeks daily single oral dose ofNevirapine (NVP) or Zidovidinerecommended for newbornsto HIV positive mothers. Maternal optimal adherence to these anti-retroviral prophylaxis regimens is imperative to prevention of vertical transmission of HIV. Limited studies investigated maternal adherence to infant's NVP prophylaxis regimen and associated factors. This study examined the level and factors influencing maternal adherence to infant's NVPprophylaxis regimenat six weeks of age. Maternal adherence to infant's NVP regimen was defined as dispensation of $95 \%$ of the total daily single dose of NVPsuspension to infants at 6 weeks of age. Maternal adherence, socio-demographic and psychosocial factors were measured using a pre-tested questionnaire. Bivariate analysis and binary logistic regression model were used to determine the level and factors influencing maternal adherence to infant's NVP regimen respectively. Results showed that $77 \%$ of the mothers had optimal adherence. Maternal age $\geq 20$ years and believe in the effectiveness of NVP in prevention of vertical transmission of HIV were associated with increased likelihood of adherence to infants NVP regimen (OR 6.18; 95\% CI: 1.64-23.24) and (OR 12.69; 95\% CI 3.07-52.44) respectively.In conclusion maternal maturity and perceived benefits of NVP seems to be important positive drivers of optimal adherence. This suggests the importance of considering psychosocial and demographic characteristics of mothers in policy and intervention designing.
\end{abstract}

Keywords: Adherence, Prophylaxis, Postpartum Period, Mothers

\section{Introduction}

Despite of advances in treatment and prevention, Human Immune Virus (HIV) /Acquired Immune Deficiency syndrome (AIDS) remains a major cause of morbidity and mortality among children and adults in sub-Saharan Africa [1]. An estimated 1.8 million children $<15$ years were living with HIV in 2017 and five thousand new infections occurred daily in both children and adults while $66 \%$ of thesenew infections occurred in sub-Saharan Africa [1]. Although estimates of new cases of HIV infection among children 0-14 years declined from 18,000 to 7600 cases, and deaths from
13,000 to 3800 cases in 2017,approximately 95,000 children in Uganda were reported to beliving with HIV/AIDSin 2017 [1]. With the increasing number $(480,000$ in 2005 to 750,000 in 2017) of women of reproductive age living with HIV/AIDS in Uganda, the increase in the incidence of HIV/AIDS among children cannot be ruled-out $[1,2]$. Of the 112,909 babies born to women living with HIV in Uganda, only $38 \%$ of were reported to have receivedNevirapine (NVP) prophylaxis, mainly attributed to prevention of mother-to-child (PMTCT) services access limitation and weakness in monitoring and evaluation system [3].The low coverage of HIV prophylaxis among mother-infant dyad 
increases the risk of verticaltransmission and impedes achievement of 2030 global goal of EMTCT [4, 5].

Existing evidence has showed that most children acquire HIV from their infected mothers. In the absence of maternal antiretroviral drug treatment (ART), infection rates among children can be as high as $15-45 \%$ [5], but NVP has been shown to reduce the risk of vertical transmission of HIV to infants from birth to 6 months but 54\% [6]. This risk reduction in vertical transmission of HIV/AIDS varying from $49 \%$ in developing countries to $100 \%$ in developed countries [7]. Maternal ART and infant NVP prophylaxis have proven efficacy and effectiveness in prevention of vertical transmission of HIV [8]. To eliminate mother-to-child transmission of HIV, WHO recommended treatment of HIV positive women and HIV prophylaxis for infantsaged 06weeks [9, 10]. Adherence to medication also known as compliancewithmedication is the extent to which a person follows physician prescribed instructions for medication intake and includes timely use of medicines in correct dose [11]. Adherence to medication can be measured by direct and indirect methods $[11,12]$. WHO recommends use of single daily dose of either Zidovudine or NVP suspensionfor HIV prophylaxis in newborns up to 4-6 weeks oflife [13]. These guidelines were adapted by Ugandan Ministry of Health and only NVP suspension is used in the Ugandan context [14].

Factors influencing adherence to medication are embedded in the components of the healthcare system and socio-cultural environment in which individuals live and work. A systematic review of both qualitative and quantitative studies on barriers and facilitators of ART adherence among HIV positive adults found that poor knowledge of HIV/AIDS \& PMTCT services, ART and its associated demands negatively affects adherence to ART [15]. A Ugandan cross-sectional study found high levels of maternal non-adherence to ART and concluded that younger age, low parity, lower travel costs were positively associated maternal adherence, however quality of statistical analysis made the validity of the results questionable [16]. Disclosure of HIV/AIDS status to a spouse and the ART treatment support provided by spouse to women tend to improve ART adherence, and retention in ART program, however fear of negative consequences women might experience from the spouse was found to be barrier to adherence to ART [15].In a South African cross-sectional study that used reported maternal adherence to infants prophylaxis regimen at six weeks postpartum, maternal adherence to ART prior to delivery was associated with $80 \%$ reduction in non-adherence to infant's NVP prophylaxis. Similarly a study conducted in Zanzibar also found that alcohol consumption, drug abuse, lack of treatment support, lack of HIV/AIDS status disclosure, drug abuses and twice a day frequency of ARV intake were all positively associated with poor adherence to ARVsamong men and women [17]. Maternal depression was also found to be associated with $4 \%$ increase in maternal non-adherence to infants NVP prophylaxis regimen [18]. A cohort study conducted in HIV clinics found that mothers that experienced intimate partner violence were less likely to adhere to their
ART regime [19]. On the contrary a cross-sectional study in Zambiaand another in South Africa found no association between maternal decision making autonomy and adherence to infant prophylaxis $[20,21]$. In addition, a Cameroonian qualitative therapeutic itineraries study of barriers or impediments to ART adherence were found to include financial constraints, limited social support and limited information on HIV/AIDS and treatment [22].

Social support defined as "the perception or experience that one is loved and cared for by others, esteemed and valued, and part of a social network of mutual assistance and obligations"[23] plays an important role in adherence to medication as such, it has been shown that social and psychosocial interventions have been shown to be effective in enhancing PMTCT service delivery and promotes retention of clients to existing services [24]. HIV positive women normally face stigmatization from inner self, friends and family members [25]. This is thought to negativelyaffect optimal adherence to infants NVP prophylaxis regimen.Emotional avoidance by Healthcare providers was found to be associated non- adherence to ART [26]. Some studies also found that patient adherence to medication may mediate the relationship between social support and health [27]. Indeed, support from friends and family can promote patient adherence as it buffers the stress associated with the illness, encourages optimism, reduces depression, and improves health behaviors [28].

Published literature on factors influencing maternal adherence to infant NVP prophylaxis regimen are scarce in Uganda. The existing literature is mainly on adherence to pediatricARTfor HIV infected children below 12 years of age $[29,30]$, and the other published literatureis on the effect of strategies applied in administrationnevirapine prophylaxis to mother-infant dyad [31] and a qualitative study that assessed maternal adherence to initiation and continuation of own ART which found that fear of HIV status disclosure, drug side effects, HIV stigma were barriers to ART initiation and adherence [32]. Effective PMTCT requires maternal adherence to her own ART, and that of her infant NVP prophylaxis [33]. This study was therefore aimed at determining the prevalence and factors that influencematernal adherence to infant's NVP prophylaxis regimen in Mulago National Hospital.

\section{Methods and Materials}

\subsection{Study Design, Data Collection and Setting}

This was a cross-sectional study conducted in Mulago national referral hospital's directorate of obstetrics and gynecology located at Kawempe division of Kampala City, Uganda. The department provides a wide-range of maternal and child healthcare services including pre-natal, natal, postnatal care, EMTCT, immunization, family planning, management of gynecological conditions and diseases. On average, the department conducts 33,000 deliveries per year with approximately 2800 babies estimated to be at risk of 
getting HIV through mother-to-child transmission [34].

\subsection{Study Population}

The study population comprised 148 mother-infant pair at six weeks post-partum with infants enrolled for 6 weeks NVPprophylaxis. A sample size of 384 was calculated using Cochran WG (1963)formula

$$
\mathrm{n}=\frac{\mathrm{z} 2 \mathrm{p} \mathrm{q}}{\mathrm{d} 2}
$$

Where $\mathrm{n}$ is the sample size, $\mathrm{Z}^{2}$ is the desired confidence level $(95 \%)=1.96, \mathrm{~d}$ is the desired level of precision set at $5 \%, \mathrm{p}$ is the estimated level of adherence to infants NVP regimen set at $50 \%$, and $\mathrm{q}$ is $1-\mathrm{p}$.Mothers who met the inclusion criteria but were unable to communicate either in English language or Luganda and those whose babies were ill were not interviewed and therefore were excluded from the study. Data was collected by trained research assistants who spoke both English and Luganda languages and had midwifery educational background. A pre-tested questionnaire developed based on a standardized questionnaire [12] was dispensed to mothers who were identified to be HIV positive through their infants immunization cards on which the acronym NVP, meaning child on NVP prophylaxis. To reduce information bias, the questionnaire was translated from English language to Luganda by a certified language specialist from Makerere University Institute of language and collected data was spot cross-checked for accuracy by the principle investigator and anomalies were corrected before the mother left the hospital premise.

\subsection{Outcome}

Maternal optimal adherence to physician prescribed daily single dose infant NVP prophylaxis regimen at 6 weeks of age, based on WHO recommendation [35] was used as the outcome variable.Self-reportedmaternal adherence to infant'sNVP prophylaxis regimenwas defined as successful completion of $95 \%$ (approximately 40 doses) of the total 42 daily single doses of NVP suspension that infantsare expected to have received by 6 weeks of age. By answering the question; "How many times did you forget to give your baby NVP since you gave birth? Mothers stated the number of doses they did not dispense to their infants. The exact number of doses dispensed at 6 weeks of age was then calculated by subtracting the total number of doses mothers forgot to dispense to their infants from birth to 6 months of age [35]. Mothers who administered $95 \%$ of the expected total of 42 single daily doses to their infants at 6 weekswere considered to have had optimal adherence and those that dispensed less than $95 \%$ ( $\leq 40$ daily doses) of the required 42 doses were considered to be non-adherent.

\subsection{Exposures}

The exposure variables collectively consisted of selfreported maternal socio-demographic and psychosocial characteristics. Maternal socio-demographic characteristics whose effect on maternal optimal adherence to infants NVP regimen were examined included maternalage groups of 1519years \& 20-49 years with earlier as the reference, marital status (categorized as single, married, cohabiting, divorced/widowed),religion categorized into either Muslims or Christians, tribe grouped into Muganda and other tribes, number of children ever born, and duration of maternal ART. Maternal psychosocial characteristics were also assessed and these included; stigma assessed by asking whether people have ever avoided the mother after having known her HIV status? Or whether she was treated badly or whether people gossip her for being HIV positive?. Maternal disclosure of her HIV positive status to others was assessed by a) whether mother told anyone she was giving her baby NVP syrup, b) whether she has ever told anyone about her HIV positive status? Mothers were also asked whether they hide away from people when giving her baby NVP. To determine social support, mothers were asked four questions; whether they had someone that provided support or reminded them to give their baby NVP syrup?, whether they had someone who takes them to a hospital in case they fell sick? Whether they had someone they could talk to them if they had sickness related to HIV or if their baby was sick? Finally their confidence in the effectiveness of NVP in prevention of HIV was assessed by asking them; whether they think NVP was effective in preventing their baby from contracting HIV from them. The questions for assessing maternal HIV psychosocial characteristics were selected from the Berger stigma scale [36] and a validated social support questionnaire [37].

\section{Statistical Analysis}

Statistical analysis was conducted to determine the distribution of maternal socio-demographic and psychosocial characteristics, level of maternal adherence and factors that influence maternal adherence to infants NVP prophylaxis regimen. To determine the level of maternal adherence to infant's NVP prophylaxis regimen by socio-demographic and psychosocial characteristics, a bivariate analysis was performed and chi-square test was used to determine proportional difference in the distribution of maternal adherence to infants NVP regimen. To determine the independent relationship between socio-demographic characteristics, psychosocial factors and maternal adherence to infant's NVP prophylaxis regimen, unadjusted and adjusted binary logistic regression models were fitted.The levels of maternal adherence to infant's NVP prophylaxis regimen were presented in proportions, odds ratios and their corresponding $95 \%$ confidence intervals were used to determine the association between socio-demographic and psychosocial characteristics and maternal adherence to infant's NVP prophylaxis regimen. Analysis was conducted using SPSS version 24.

\section{Ethical Considerations}

Ethical approval of the study was granted by the School of 
Health Sciences Research and Ethics Committee (REC) Board (SHSREC 2017-009). A written informed consent was also obtained from each study participant. Confidentiality of information provided by the study participants was maintained right from interview room where no one except the participant interacted with the interviewer. Each study participant was assigned a serial number to avoid identification and filled-in questionnaires were kept understrict custody of the principle investigator in a locked cabin. Data entry into SPSS statistical software was also done by the principle investigator and questionnaires were destroyed after the data entry and analysis.

\section{Results}

Characteristics of the study population

As shown on Table 1, a total of 148 mother-infant pair was analyzed in this study. Themean age of mothers was 24.5( $\mathrm{SD} \pm 1.8)$ years, withaminimum and maximum age of 15 and 42 years respectively. Half of the mothers were Baganda by tribe. Majority (77\%) of themwere Christians in different denominations, with Muslims comprising of $23 \%$ of the study population. Most (69\%) of the mothers were cohabiting and only $20 \%$ were married. More than half (52.7\%) knew their HIV status more than a year before the date of the interview. More than half $(56.1 \%)$ of the mothers were on ART for 6-12 months, $56.8 \%$ of them delivered at least one child after HIV diagnosis. Regarding maternal psycho-social characteristics, $71.6 \%$ of the mothers weren't avoided by people for being HIV positive, only $26.4 \%$ were experienced mistreatment of any form by people for their being HIV positive, $24.3 \%$ reported being gossiped by colleagues and friends of their HIV status, more than half $(52.7 \%)$ of the mothers disclosed to colleagues and friend giving NVP suspension to their infants, and majority $84.5 \%$ of them reported hiding themselves when giving NVP to their infants. Majority $(66.2 \%)$ of the mothers also reported having someone to remind them to give NVP syrup to their infants. More than half( $62.2 \%)$ of the mothers had someone to take care of them when either the infant or they themselves were sick and $67.6 \%$ of the women had someone to guide them if they experienced HIV related health problem. With regards to maternal perception of NVPandtherisk of infant getting HIV, majority of the mothers believedNevirapine was effective in preventing HIV and close to half (45.3\%) of believed their infants were at risk of getting HIV from them.

Maternal level of adherence to infant's $\mathrm{N}$ prophylaxis regimen.

Table 1 also shows the level of maternal adherenceto infants NVP prophylaxis regimen by socio-demographic characteristics and psychosocial experiences. Adherence was assessed when infants were at 6 weeks of age.101 (77\%) of the women were adherent to infant's NVP prophylaxis regimen i.e. they reported having given their infants $95 \%$ of the expected 42 daily doses of NVPsuspension. When maternal adherence to infants NVP prophylaxis regimen wasdisaggregated by socio-demographic characteristics and psychosocial experiences, adherence level among women aged 20-49 years was $84.9 \%$ higher than for those less than 20 years $(44.80 \%)$. Adherence levels were also above $70 \%$ for women in all categories of marital status and tribe. $79.8 \%$ and $67.6 \%$ of Christian and Muslim women were adherent to infant NVP prophylaxis. Across ART treatment duration categories, the levels of maternal adherence to NVP prophylaxis regimens were above $70 \%$. Likewise $80.7 \%$ of the women who had more than one live child were adherent and $66.70 \%$ of those with only one child were equally adherent to NVP prophylaxis. Levels of maternal adherence to infant NVP prophylaxis regimen across all categories of psychosocial experiences were also above $60 \%$. When mothers were asked of their perception of risk of their infant getting HIV, those that perceived that their infants were not risk of getting HIV had $82 \%$ adherence and those that perceived that their infants weren't at risk of getting HIV were $72.8 \%$ adherent. Regarding their believe about the effectiveness of NVP in prevention of vertical transmission of HIV, mothers who believed NVP was effective in prevention of vertical transmission of HIV were $84.9 \%$ adherent and those that believed NVP was ineffective had $31.8 \%$ level of adherence.

Factors influencing maternal adherence to infant NVP prophylaxis regimen

Table 2 shows the crude and adjusted odds ratios (OR) and their corresponding 95\% Confidence Intervals (CI) for maternal adherence to NVP by socio-demographic characteristics and psychosocial experiences. Compared to women aged 15-19 years, those aged 20-42 years were more likely to report adherence to infants NVP prophylaxis regimen (OR 6.18; 95\% CI 1.64 -23.24). Baganda women compared to women from other tribes were less likely to be adherent to infants NVP prophylaxis regimen (OR 0.66; CI 0.21-1.99). When the likelihood of maternal adherence according to marital status was assessed, single mothers, divorced/widows and cohabiting women were also more likely to report being adherent to infants NVP prophylaxis regimen (OR 1.91; CI 0.21-16.77), (or 1.53 CI 0.19-12.43), (OR 1.24; CI 0.21-07.55) respectively. Compared to women who had only one living child, those with two or more children were less likely to adhere to NVP prophylaxis regimen (OR 0.48; 0.11- 2.03) had high likelihood of adherence than married women and was so was the divorced/widowed, cohabiting women. Mothers who were on ART for more than a year were less likely be adherent to NVP prophylaxis (OR 0.86; CI 0.25-2.96), mothers who were badly treated by colleagues/friends because of their HIV status were more likely to adhere to NVP prophylaxis (OR 3.98; 0.12-130.74). Mothers who reported having been gossiped by people because of being HIV positive were more likely to adhere to NVP prophylaxis (OR 1.02; 0.09-12.05), those not avoided by people because oftheir HIV status also less likely to adhere to NVP prophylaxis (OR 0.53; CI 0.0310.18). Those that shared information on giving NVP to their infants were less likely to adhere to NVP (OR 0.43 ; 0.14 1.34). Mothers who had someone to remind them to give 
NVP to infants were also less likely to adhere to NVP prophylaxis (OR 0.88; CI 0.13-6.12), Mothers that do not hide away when giving NVP to infants were more likely be to adherent than those that hideaway (OR 6.00 (0.91-39.49). those that had someone to take care of them or baby when sick were however less likely to be adherent to NVP (OR
0.34 CI; 0.06-1.99). Mother who thought their infantswere at risk of getting HIV were more likely to be adherent to NVP than those that did not (OR 1.49; CI 0.44-5.04) and lastly those that believed NVP is effective in prevention of HIV infection more likely to be adherent to infants NVP (OR 12.69; CI 3.07-52.44)

Table 1. The distribution of socio-demographic and psychosocial factors by maternal adherence and non-adherence to infant's NVPprophylaxis regimen.

\begin{tabular}{|c|c|c|c|}
\hline \multirow{2}{*}{ Maternal Characteristics } & \multicolumn{3}{|c|}{ Maternal Adherence } \\
\hline & Total n (\%) & Adherent n (\%) & Non-Adherent n (\%) \\
\hline \multicolumn{4}{|l|}{ Maternal Age (Years) } \\
\hline $15-19$ & $029(19.60)$ & $013(44.80)$ & $16(55.20)$ \\
\hline $20-42$ & $119(80.40)$ & $101(84.90)$ & $18(15.10)$ \\
\hline Total & $148(100)$ & $114(77.00)$ & $34(23.00)$ \\
\hline \multicolumn{4}{|l|}{ Marital Status } \\
\hline Married & $20(13.50)$ & $24(77.40)$ & $04(20.00)$ \\
\hline Divorced/Widow & $28(18.90)$ & $21(75.00)$ & $07(25.00)$ \\
\hline Single mother & $31(20.90)$ & $24(77.40)$ & $07(22.60)$ \\
\hline Cohabiting & $69(46.60)$ & $53(76.80)$ & $16(23.20)$ \\
\hline \multicolumn{4}{|l|}{ Religion } \\
\hline Christian & $114(77.00)$ & $91(79.80)$ & $23(20.20)$ \\
\hline Muslim & $34(23.00)$ & $23(67.60)$ & $11(32.40)$ \\
\hline \multicolumn{4}{|l|}{ Tribe } \\
\hline Muganda & $74(50.00)$ & $57(77.00)$ & $17(23.00)$ \\
\hline Others & $74(50.00)$ & $57(77.00)$ & $17(23.00)$ \\
\hline \multicolumn{4}{|l|}{ Years on HIV/AIDS Treatment } \\
\hline 6-12 months & $83(56.10)$ & $62(74.70)$ & $21(25.30)$ \\
\hline$\geq 1$ year & $65(43.90)$ & $52(80.00)$ & $13(20.00)$ \\
\hline \multicolumn{4}{|c|}{ Total Number of ever born children } \\
\hline 1 child & $039(26.40)$ & $26(66.70)$ & $13(33.30)$ \\
\hline$>1$ child & $109(73.60)$ & $88(80.70)$ & $21(19.30)$ \\
\hline \multicolumn{4}{|c|}{ Live deliveries after HIV diagnosis } \\
\hline 1 child & $84(56.80)$ & $58(69.00)$ & $26(31.00)$ \\
\hline Above 1 child & $64(43.20)$ & $56(87.50)$ & $08(12.50)$ \\
\hline \multicolumn{4}{|c|}{ Mother avoided by colleagues b'seof HIV Status } \\
\hline No & $106(71.60)$ & $85(80.20)$ & $21(19.80)$ \\
\hline Yes & $042(28.40)$ & $29(69.00)$ & $13(31.00)$ \\
\hline \multicolumn{4}{|l|}{ Treated badly by associates } \\
\hline No & $109(73.60)$ & $88(80.70)$ & $21(19.30)$ \\
\hline Yes & $039(26.40)$ & $26(66.70)$ & $13(33.30)$ \\
\hline \multicolumn{4}{|c|}{ Colleagues and Friends gossip about mothers HIV status } \\
\hline No & $112(75.70)$ & $91(81.20)$ & $21(18.80)$ \\
\hline Yes & $036(24.30)$ & $23(63.90)$ & $13(36.10)$ \\
\hline \multicolumn{4}{|c|}{ Mother disclosed giving NVP syrup to child to colleagues } \\
\hline No & $70(47.30)$ & $46(65.70)$ & $24(34.30)$ \\
\hline Yes & $78(52.70)$ & $68(87.20)$ & $10(12.80)$ \\
\hline \multicolumn{4}{|c|}{ Mother hides when giving her infant NVP Syrup } \\
\hline No & $022(15.00)$ & $19(86.40)$ & $03(13.60)$ \\
\hline Yes & $125(85.00)$ & $94(75.20)$ & $31(24.80)$ \\
\hline \multicolumn{4}{|c|}{ Mother disclosed HIV status to someone } \\
\hline No & $58(39.20)$ & $43(74.10)$ & $15(25.90)$ \\
\hline Yes & $90(60.80)$ & $71(78.90)$ & $19(21.10)$ \\
\hline \multicolumn{4}{|c|}{ Mother has someone to remind her to give NVP syrup } \\
\hline No & $50(33.80)$ & $33(66.00)$ & $17(34.00)$ \\
\hline Yes & $98(66.20)$ & $81(82.70)$ & $17(17.30)$ \\
\hline \multicolumn{4}{|c|}{ Mother has someone to take care of her when sick or baby sick } \\
\hline No & $56(37.80)$ & $45(80.40)$ & $11(19.60)$ \\
\hline Yes & $92(62.20)$ & $69(75.00)$ & $23(25.00)$ \\
\hline \multicolumn{4}{|c|}{ Mother has someone to guide her if she has HIV related problem } \\
\hline No & $048(32.40)$ & $29(60.40)$ & $19(39.60)$ \\
\hline Yes & $100(67.60)$ & $85(85.00)$ & $15(15.00)$ \\
\hline Mother thinks baby at risk $o$ & & & \\
\hline No & $81(54.70)$ & $59(72.80)$ & $22(27.20)$ \\
\hline Yes & $67(45.30$ & $55(82.10)$ & $12(17.90)$ \\
\hline Mothers thinks NVP is effec & HIV & & \\
\hline No & $022(14.90)$ & $007(31.80)$ & $15(68.20)$ \\
\hline Yes & $126(85.10)$ & $107(84.90)$ & $19(15.10)$ \\
\hline
\end{tabular}


Table 2. Crude and adjusted odds ratio and 95\% confidence intervals (CI) for maternal adherence to infant'sNVP prophylaxis regimen by socio-demographic and psychosocial characteristics.

\begin{tabular}{|c|c|c|}
\hline Maternal Characteristics & Crude OR (95\% CI) & Adjusted OR (95\% CI) \\
\hline \multicolumn{3}{|l|}{ Age (Years) } \\
\hline $15-19$ & 1.00 & 1.00 \\
\hline $20-49$ & $6.91(2.84-16.77)$ & $6.18(1.64-23.24)$ \\
\hline \multicolumn{3}{|l|}{ Tribe } \\
\hline Others & 1.00 & 1.00 \\
\hline Muganda & $1.00(0.47-02.15)$ & $0.66(0.21-01.99)$ \\
\hline \multicolumn{3}{|l|}{ Marital Status } \\
\hline Married & 1.00 & 1.00 \\
\hline Single mother & $1.21(0.35-4.13)$ & $1.91(0.21-16.77)$ \\
\hline Divorced/Widow & $1.04(0.38-2.84)$ & $1.53(0.19-12.43)$ \\
\hline Cohabiting & $0.91(0.33-2.52)$ & $1.24(0.21-07.55)$ \\
\hline \multicolumn{3}{|l|}{ Religion } \\
\hline Christian & 1.00 & 1.00 \\
\hline Muslims & $0.53(0.23-1.24)$ & $0.62(0.18-2.09)$ \\
\hline \multicolumn{3}{|c|}{ Total Number of children ever born } \\
\hline 1 child & 1.00 & 1.00 \\
\hline$\geq 1$ child & $3.14(1.31-7.52)$ & $0.48(0.11-2.03)$ \\
\hline \multicolumn{3}{|c|}{ Mothers during on HIV/AIDS Treatment } \\
\hline 6-12 months & 1.00 & 1.00 \\
\hline Above one year & $1.36(0.62-2.97)$ & $0.86(0.25-2.96)$ \\
\hline \multicolumn{3}{|c|}{ People avoided mother b'se of HIV/AIDS status } \\
\hline Yes & 1.00 & 1.00 \\
\hline No & $0.15(0.25-1.24)$ & $0.53(0.03-10.18)$ \\
\hline \multicolumn{3}{|c|}{ Mother treated badly by colleagues/friend } \\
\hline No & 1.00 & 1.00 \\
\hline Yes & $0.48(0.21-1.08)$ & $3.98(0.12-130.74)$ \\
\hline \multicolumn{3}{|c|}{ People gossip about mothers HIV status } \\
\hline No & 1.00 & 1.00 \\
\hline Yes & $0.41(0.18-0.94)$ & $1.02(0.09-12.05)$ \\
\hline \multicolumn{3}{|c|}{ Mother shared information about giving NVP for child } \\
\hline No & 1.00 & 1.00 \\
\hline Yes & $3.55(1.55-8.11)$ & $0.43(0.14-1.34)$ \\
\hline \multicolumn{3}{|c|}{ Mother has someone to remind her of giving NVP to infant } \\
\hline No & 1.00 & 1.00 \\
\hline Yes & $2.46(1.12-5.38)$ & $0.88(0.13-6.12)$ \\
\hline \multicolumn{3}{|c|}{ Mother hides away when giving NVP to infant } \\
\hline Yes & 1.00 & 1.00 \\
\hline No & $2.09(0.58-7.54)$ & $6.00(0.91-39.49)$ \\
\hline \multicolumn{3}{|c|}{ Mother has someone to take care of her/baby when sick } \\
\hline No & 1.00 & 1.00 \\
\hline Yes & $0.73(0.33-1.65)$ & $0.34(0.06-1.99)$ \\
\hline \multicolumn{3}{|c|}{ Mother thinks baby at risk of getting HIV } \\
\hline No & 1.00 & 1.00 \\
\hline Yes & $0.59(0.27-1.29)$ & $1.49(0.44-5.04)$ \\
\hline \multicolumn{3}{|c|}{ Mother thinks NVP effect in HIV prevention } \\
\hline No & 1.00 & 1.00 \\
\hline Yes & $12.07(4.35-33.51)$ & $12.69(3.07-52.44)$ \\
\hline
\end{tabular}

All covariates were mutually adjusted, point estimates \& CI shows independent effects of each factors

\section{Discussion}

This study assessed the prevalence and factors influencing maternal adherence to NVP prophylaxis for prevention vertical transmission of HIV from mother to infants at Mulagonational referral hospital's department of obstetrics and gynecology. The findings showed high maternal adherence to infants NVP prophylaxis at 6 weeks of age. Similarly when maternal level of adherence was disaggregated according to socio-demographic and psychosocial characteristics, across all categories of disaggregation variables, the level of maternal adherence was above $60 \%$ implying institutional mechanisms established to ensure EMTCT services effectiveness are functional and efficient in service delivery. The findings of this study revealed relatively high level of optimal maternal reported adherence to infants NVP prophylaxis regimenthan thosereported in a Kenyan and Namibian studies [25,38] and similar to those in studies conducted in Sub-Saharan African countries [12] and correspondingly similar to findings of a Uganda study conducted among HIV positive adults in an urban ambulatory Clinic in Kampala [39]. Sub-optimal maternal adherence levels was $23.0 \%$,which is much higher than the acceptable level of $<5 \%$ [35]. This might therefore endanger the fight against vertical transmission of HIV, 
increase HIV/AIDs disease burden among children and increasehealth care costs especially among affected HIV infected children and families and Uganda at large [40].

Regarding factors that positively influenced maternal level of adherence to infants NVP prophylaxis regimen dispensation from 0-6 weeks, this study found maternal age and believe about the effectiveness of NVP in prevention of vertical transmission of HIV associated with maternal adherence. Specifically, relatively older mothers (20-49) years of age were found to have higher likelihood of adherence than their counterparts who were aged 15-19 years. This might be due to the possibility that older mothers have accumulated more health knowledge than their younger counterparts, therefore have more HIV/AIDS illness experience and better cues to action. Similarly women at higher birth orders might be overwhelmed by numbers of children that tends to increase female gender responsibilities in African context and interfere with adherence to maternal adherence to infants NVP prophylaxis regimen.Likewise mothers that believed NVP was effective in prevention of vertical transmission of HIV might be compelled to dispense NVP suspension to their infants because of their level of confidence the drug's effectiveness as well as in the fear of their infants getting HIV infection from them. Pender's Health Promotion Model (2006) emphasizes that full understanding of the benefits and outcome of a health promoting action instills motivation and the desire to embark on a health promoting behavior [41]. Understanding the benefits also assists the individual to positively identify barriers to a health promoting behavior then strategize ways of overcoming them in order to achieve the outcome of a health promoting behavior. It is believed that when HIV positive womenperceive that the NVP syrup is effective in PMTCT they are most likely to adhere to its administration to their HIV exposed babies [42] which reflects findings from studies which showed that perceived benefit from medication promotes adherence [43, 44]. Maternal marital status was also found to have an effect on maternal adherence to infants NVP prophylaxis regimen. This might be attributed to domestic violence experienced by married mothers as their husbands might blame them for having infected them with the HIV virus, hence denial of responsibility that has consequential effects on maternal adherence to infants NVP prophylaxis regimen. Mothers that experienced bad treatment by acquaintances for their HIV status were also more likely to be adherent to infants NVP prophylaxis regimen. This might be explained by maternal hope in their child as a propagator of their life, hence their infant gives them hope and motivate them to give them maternal love before their departure from the earth hence their adherence to infants NVP prophylaxis regimen. Havingbeen gossiped about their HIV status provides cue to action for mothers. This implies that mothers were more likely to reflect on the negative consequences of their own actions or inactions that contributed to their HIV positive status and the need to maximize possibilities of their infants not acquiring the infection from them. Mothers that hide from other people when giving NVP to their infants had higher likelihood of adherence than those who were not hiding when giving NVP suspension to their infants. This might be attributed to reverse causality as well as to perceived fear of their infants getting HIV from them and their experience of the disease, its physical, social and psychological effects. Lastly mothers that believed their infants were at risk of getting HIV from them also had high likelihood of adherence to infants NVP prophylaxis regimen. This might be linked to maternal love ad desire of not letting their infant go through diseased state experience they had hence maximization of the available opportunity of keeping their infants free of HIV/AIDS.

The findings of this study also showed that women's tribal affiliation negatively influence maternal adherence to NVP prophylaxis regimen. Howeverthere wasn't adequate evidence to state so. Tribal differences with regards to medication adherence might be linked to cultural differences. It could be that the Baganda might inherently have stronger believe in the effectiveness of theirtraditional medicine as opposed to modern medicine. Similarly women who believed in the islamic faith were less likely to be adherent to NVP prophylaxis compared to christian women. Religious differences in level of maternal adherence to NVP and other medications hasnt been explicit. One might link this differences to inherent religious believes and practices. Talking of muslims in particular, the religion encourages men to marry a maximum of four wives. Muslim women might constrained by economic factors especially if their husbands have more wives and can't meet the needs of family. Hence the possibility that muslim women might need to look for ways of earning through petty business is high, leaving children at home or staying in work place where there are many people and might not dispense NVP due tofear of letting others know their HIV status. Having more than one child was also linked to less likelihood of maternal adherence to NVP compared to having only one child. Likewise mothers that were on ART for more than a year were less likely to be adherent to nevirapine prophylaxis than those that were ART for 6-12 months. This could partly be attributed to reverse causality an inherent weakness of crosssectional studies since the expectation is that mothers who were on ART might have the experience of daily intake of ARVs that could have been translated into adherence to infants NVP regimen dispensation. On the other hand we might speculate that mothers who were on ARVs might be have other factors that unexplicated factors that were not controllled for in this study. Women who had delivered more than one child since HIV diagnosis were less likely to be adherent to their infants NVP prophylaxis regimen when compared to their counterparts that had one child. Thismay be due to previous experiences and familiarity as subsequent deliveries are more likely to be in health facilities where EMTCT services are provided compared to the first time mothers $[45,46]$. Mothers who were avoided by society for their HIV status, those that shared information on giving their infants NVP, and those hideaway when giving NVP and mothers that believed their infants were at risk of getting HIV 
infection were more likely to be adherent to infants NVP prophylaxis. Likewise on the contrary, mothers that had someone to remind to give NVP suspension to their infants had low likelihood of adherence compared with mothers that did not. Receiving social support was thought to occur only if the HIV positive person discloses his or her status. Social support of people living with HIV/AIDS is important for adherence to treatment [25]. An ethnographic study conducted in three African countries to explore better adherence levels found in Africa has concluded that social support played a major part in ensuring patients' adherence to HAART [47], support from community encourages individuals to seek treatment and care. These findings are not consistent with many ARV therapy adherence studies [48, 49] where lack of social support from family and friends was found to affect adherence negatively. The social support reported in literature varied from reminding patients to take medication, actual giving out the medication, staying with the children as the person goes for appointments and/or offering food and drink to accompany the intake of medication. Without social support, it is difficult for HIV positive women to adhere to treatment and breastfeeding regimens that are necessary in reducing mother to child transmission of the disease [50].

\section{Conclusion}

Most mothers attending EMTCT services in Mulago regional hospitals department of obstetrics and gynecology achieved the optimal level of adherence to infants NVP prophylaxis regimen dispensation although a big margin remains to achieve the desired $<5 \%$ level of non-adherence. Factors that positively influenced maternal adherence to infants NVP prophylaxis regime were maternal age and believe in the effectiveness of NVP in prevention of vertical transmission of HIV. Major inhibitors of maternal adherence to infants NVP prophylaxis were psychosocial factors linked HIV stigmatization by associates and community members.Focusing on improving maternal level of confidence in the effectiveness of NVP prevention of vertical transmission of HIV and promoting factors that delay pregnancy might be relevant in the efforts towards EMTCT. Similarly, focus on efforts for promoting social support for HIV infected mothers and reducing stigmatization might be important for achievement of EMTCT goals. Based on the limited sample size that might have obscured the effect of some socio-demographic and psychosocial factors, conduct of hospital or community based studies with a larger sample size might important for ascertainment of the results of this study.

\section{Strengths and Limitations}

To the best of our knowledge, this is the first study that assessed the level of maternal adherence to infants NVP prophylaxis regimen in Uganda. Maternal adherence was measured based using maternal verbal report, hence prone to recall bias. This study was also institution based study; therefore findings cannot be generalizable to all women living either in Kampala or Uganda. Being a cross-sectional study with small sample size, reverse causation cannot be avoided and results are only indicative of the level of and factors of maternal adherence to NVP at the time of study conduct.

\section{Conflict of Interest}

The authors have no conflict of interest to declare.

\section{Acknowledgements}

We wish to acknowledge Lira University, Victoria University Kampala and Department of Nursing, Makerere University for providing enabling environment for research collaboration and the study participants for accepting and providing informed consent to take part in the study.

\section{References}

[1] UNAIDS, Global HIV \& AIDS statistics - 2018 fact sheet. 2018, UNAIDS: Geneva, Switzerland.

[2] WHO. HIV Data and statistics 2018 [cited 2018 18/8/2018]; Available from: http://www.who.int/hiv/data/en/.

[3] Uganda, A., Commission (UAC): HIV and AIDS Uganda Country Progress Report, 2013. Kampala: UAC; 2014. 2016.

[4] Vrazo, A.C., D. Sullivan, and B.R. Phelps, Eliminating mother-to-child transmission of HIV by 2030: 5 strategies to ensure continued progress. Global Health: Science and Practice, 2018. 6(2): p. 249-256.

[5] WHO. Mother -to- child transmission of HIV. 2018 [cited 2018 18/8/2018]; Available from: http://www.who.int/hiv/topics/mtct/about/en/.

[6] Coovadia, H.M., et al., Efficacy and safety of an extended nevirapine regimen in infant children of breastfeeding mothers with HIV-1 infection for prevention of postnatal HIV-I transmission (HPTN 046): a randomised, double-blind, placebo-controlled trial. The Lancet, 2012. 379(9812): p. 221228.

[7] Vreeman, R.C., et al., A systematic review of pediatric adherence to antiretroviral therapy in low-and middle-income countries. The Pediatric infectious disease journal, 2008. 27(8): p. 686-691.

[8] Flynn, P.M., et al., Prevention of HIV-1 Transmission Through Breastfeeding: Efficacy and Safety of Maternal Antiretroviral Therapy Versus Infant Nevirapine Prophylaxis for Duration of Breastfeeding in HIV-1-Infected Women With High CD4 Cell Count (IMPAACT PROMISE) A Randomized, Open-Label, Clinical Trial. JAIDS Journal of Acquired Immune Deficiency Syndromes, 2018. 77(4): p. 383-392.

[9] UPdaTE, P.J.W., Geneva, April, Use of antiretroviral drugs for treating pregnant women and preventing HIV infection in infants. 2012. 
[10] Organization, W.H., Consolidated guidelines on the use of antiretroviral drugs for treating and preventing HIV infection: recommendations for a public health approach. 2016: World Health Organization.

[11] Osterberg, L. and T. Blaschke, Adherence to medication. New England Journal of Medicine, 2005. 353(5): p. 487-497.

[12] Desmond, C. and J. Gow, Impacts and interventions: The HIV/AIDS epidemic and the children of South Africa. 2015: University of Natal Press.

[13] MOH, CONSOLIDATED GUIDELINES FOR PREVENTION AND TREATMENT OF HIV IN UGANDA 2016.

[14] Uganda, M.o.H., Uganda Clinical Guideline 2016. 2016.

[15] Hodgson, I., et al., A systematic review of individual and contextual factors affecting ART initiation, adherence, and retention for HIV-infected pregnant and postpartum women. 2014. 9(11): p. e111421.

[16] Decker, S., et al., Prevention of mother-to-child transmission of HIV: Postpartum adherence to Option B+ until 18 months in Western Uganda. 2017. 12(6): p. e0179448.

[17] Haji, H.J.I.J.o.I.D., Factors associated with poor adherence to ant-retroviral therapy among HIV/AIDS patients attending care and treatment clinic at Mnazi Mmoja Hospital, Zanzibar. 2018. 73: p. 250-251.

[18] Cook, R., et al., A Bayesian Analysis of Prenatal Maternal Factors Predicting Nonadherence to Infant HIV Medication in South Africa. 2018: p. 1-9.

[19] Yee, L.M., et al., Relationship between intimate partner violence and antiretroviral adherence and viral suppression in pregnancy. Sexual \& Reproductive Healthcare, 2018. 17: p. 711.

[20] Ford, C., et al., Women's decision-making and uptake of services to prevent mother-to-child HIV transmission in Zambia. 2018. 30(4): p. 426-434.

[21] Ford, C.E., et al., Maternal Decision-Making and Uptake of Health Services for the Prevention of Mother-to-Child HIV Transmission: A Secondary Analysis. 2018: p. 1-9.

[22] Landefeld, C., et al., Prevention of Mother-to-Child Transmission of HIV in Yaounde: Barrier to Care. 2018. 30(1): p. 116-120.

[23] Taylor, S.E., H. Friedman, and R. Silver, Social support. Foundations of health psychology, 2007: p. 145-171.

[24] Ambia, J. and J.J.J.o.t.I.A.S. Mandala, A systematic review of interventions to improve prevention of mother - to - child HIV transmission service delivery and promote retention. 2016. 19(1): p. 20309.

[25] Musenjeri, S.K., Factors affecting adherence to treatment in HIV exposed infants in Mumias region, Western Kenya. 2016, COHES, JKUAT.

[26] Berghoff, C.R., et al., The Role of Emotional Avoidance, the Patient-Provider Relationship, and Other Social Support in ART Adherence for HIV+ Individuals. AIDS and Behavior, 2018. 22(3): p. 929-938.

[27] Dunbar-Jacob, J., E. Schlenk, and M. McCall, Handbook of health psychology. 2001, Mahwah: Erlbaum.
[28] DiMatteo, M.R., Social support and patient adherence to medical treatment: a meta-analysis. 2004, American Psychological Association.

[29] Haberer, J.E., et al., Multiple measures reveal antiretroviral adherence successes and challenges in HIV-infected Ugandan children. PloS one, 2012. 7(5): p. e36737.

[30] Olds, P.K., et al., Assessment of HIV antiretroviral therapy adherence by measuring drug concentrations in hair among children in rural Uganda. AIDS care, 2015. 27(3): p. 327-332.

[31] Karcher, H., et al., Outcome of Different Nevirapine Administration Strategies in Preventin g Mother-to-Child Transmission (PMTCT) Programs in Tanzania and Uganda. Journal of the International AIDS Society, 2006. 8(2): p. 12.

[32] Buregyeya, E., et al., Facilitators and barriers to uptake and adherence to lifelong antiretroviral therapy among HIV infected pregnant women in Uganda: a qualitative study. BMC pregnancy and childbirth, 2017. 17(1): p. 94.

[33] UNAIDS, Uganda Global AIDS Response Report 2014. 2015.

[34] MJAP, fast tracking towards 90:90:90 Makerere University Joint AIDS Programme, Annual report 2015. 2015.

[35] National AIDS Control Organisation, Pediatric Antiretroviral Therapy (ART) Guidelines. 2013.

[36] Berger, B.E., C.E. Ferrans, and F.R. Lashley, Measuring stigma in people with HIV: Psychometric assessment of the HIV stigma scale $\uparrow$. Research in nursing \& health, 2001. 24(6): p. 518-529.

[37] Sarason, I.G., et al., A brief measure of social support: Practical and theoretical implications. Journal of social and personal relationships, 1987. 4(4): p. 497-510.

[38] Okawa, S., et al., Longitudinal adherence to antiretroviral drugs for preventing mother-to-child transmission of HIV in Zambia. BMC pregnancy and childbirth, 2015. 15(1): p. 258.

[39] Ngabirano, T., et al., Psychosocial Adaptation and ART Adherence of HIV-infected Adults at an Urban Ambulatory Clinic in Uganda. J Psychol Psychother, 2016. 6(236): p. 2161-0487.10002.

[40] Nachega, J.B., et al., Association of antiretroviral therapy adherence and health care costs. Annals of internal medicine, 2010. 152(1): p. 18-25.

[41] Srof, B.J. and B. Velsor-Friedrich, Health promotion in adolescents: A review of Pender's health promotion model. Nursing Science Quarterly, 2006. 19(4): p. 366-373.

[42] Ekama, S., et al., Pattern and determinants of antiretroviral drug adherence among Nigerian pregnant women. Journal of pregnancy, 2012. 2012.

[43] Holstad, M.K.M., et al., Factors associated with adherence to antiretroviral therapy. Journal of the Association of Nurses in AIDS Care, 2006. 17(2): p. 4-15.

[44] Luszczynska, A., Y. Sarkar, and N. Knoll, Received social support, self-efficacy, and finding benefits in disease as predictors of physical functioning and adherence to antiretroviral therapy. Patient Education and Counseling, 2007. 66(1): p. 37-42. 
[45] Teferra, A.S., F.M. Alemu, and S.M. Woldeyohannes, Institutional delivery service utilization and associated factors among mothers who gave birth in the last 12 months in Sekela District, North West of Ethiopia: A community-based cross sectional study. BMC pregnancy and childbirth, 2012. 12(1): p. 74.

[46] Montagu, D., et al., Where do poor women in developing countries give birth? A multi-country analysis of demographic and health survey data. PloS one, 2011. 6(2): p. e17155.

[47] Ware, N.C., et al., Explaining adherence success in subSaharan Africa: an ethnographic study. PLoS medicine, 2009. 6(1): p. e1000011.
[48] Remien, R.H., et al., Adherence to medication treatment: A qualitative study of facilitators and barriers among a diverse sample of HIV+ men and women in four US cities. AIDS and Behavior, 2003. 7(1): p. 61-72.

[49] Vervoort, S.C., et al., Adherence in antiretroviral therapy: a review of qualitative studies. Aids, 2007. 21(3): p. 271-281.

[50] Walcott, M.M., et al., Facilitating HIV status disclosure for pregnant women and partners in rural Kenya: a qualitative study. BMC public Health, 2013. 13(1): p. 1115. 